\title{
Unnormalized nonextensive expectation value and zeroth law of thermodynamics
}

\author{
Qiuping A. Wang \\ Institut Supérieur des Matériaux du Mans, 44, Av. Bartholdi, 72000 Le Mans, France \\ awang@ismans.univ-lemans.fr
}

\begin{abstract}
We show an attempt to establish the zeroth law of thermodynamics within the framework of nonextensive statistical mechanics based on the classic normalization $\operatorname{Tr} \hat{\rho}=1$ and the unnormalized expectation $x=\operatorname{Tr} \hat{\rho}^{q} \hat{x}$. The first law of thermodynamics and the definition of heat and work in this formalism are discussed.
\end{abstract}

02.50.-r,05.20.-y,05.30.-d,05.70.-a 


\section{INTRODUCTION}

A basic assumption of the equilibrium thermodynamics is the existence of equilibrium state between two systems $A$ and $B$ at which $T(A)=T(B)$ and $f(A)=f(B)$, where $T$ is the absolute temperature and $f$ the generalized force (pressure, chemical potential, electro-magnetic field, etc.), respectively. This is the so called zeroth law of thermodynamics. Up to now, no empirical evidence shows the contrary. So it is assumed that all thermodynamic theories addressing systems at equilibrium should conform with this law.

Maxwell-Boltzmann statistics $(M B S)$ gives a beautiful statistical interpretation of the zeroth law through Gibbs entropy $S=-k \operatorname{Tr} \hat{\rho} \ln \hat{\rho}$ and Maxwell-Boltzmann distribution $\hat{\rho}=\frac{1}{Z} e^{-\beta\left(\hat{H}-\hat{f}_{i} \hat{x}_{i}\right)}$ for a given ensemble in the representation of energy $\hat{H}$ and of the external variable $\hat{x}_{i}\left(\hat{x}_{i}\right.$ can be volume, particle number, etc), where $\beta=1 / k T$ and $Z=\operatorname{Tr} e^{-\beta\left(\hat{H}-f_{i} \hat{x}_{i}\right)}$. Gibbs entropy can be recast as $S=k \ln Z+k \beta E-k \beta f_{i} x_{i}\left(E=\operatorname{Tr} \hat{\rho} \hat{H}\right.$ and $\left.x_{i}=\operatorname{Tr} \hat{\rho} \hat{x}_{i}\right)$ where the term with double index $f_{i} x_{i}$ signifies a summation over $i$. In this formalism, a variation of the entropy of the total system $A+B$ is written as

$$
\begin{aligned}
d S(A+B) & =\frac{\partial S(A)}{\partial E(A)} d E(A)+\frac{\partial S(B)}{\partial E(B)} d E(B)+\frac{\partial S(A)}{\partial x_{i}(A)} d x_{i}(A)+\frac{\partial S(B)}{\partial x_{i}(B)} d x_{i}(B) \\
& =k\left[\beta(A) d E(A)+\beta(B) d E(B)+\beta(A) f_{i}(A) d x_{i}(A)+\beta(B) f_{i}(B) d x_{i}(B)\right] \\
& =0
\end{aligned}
$$

because we suppose $S(A+B)=S(A)+S(B)$ and $d S(A+B)=0$ at equilibrium in the total system. Considering that $d E(A+B)=d[E(A)+E(B)]=d E(A)+d E(B)=0$ and $d x_{i}(A+B)=$ $d\left[x_{i}(A)+x_{i}(B)\right]=d x_{i}(A)+d x_{i}(B)=0$, we get $\beta(A)=\beta(B)$ and $f_{i}(A)=f_{i}(B)$.

This empirical law was believed [1] to be absent within the nonextensive statistical mechanics $(N S M)$ proposed by Tsallis and co-workers [2 [5]. Recently, some authors show that it can be established within $N S M$ by, respectively, the approach with the standard normalization $\operatorname{Tr} \hat{\rho}=1$ and the normalized expectation $x=\operatorname{Tr} \hat{\rho}^{q} \hat{x} / \operatorname{Tr} \hat{\rho}^{q}$ by neglecting the nonextensive correlation term in $\hat{H}$ (assuming $E(A+B)=E(A)+E(B)$ ) [11], and the approach of incomplete normalization $\operatorname{Tr} \hat{\rho}^{q}=1$ with the normalized expectation $x=\operatorname{Tr} \hat{\rho}^{q} \hat{x}$ in keeping the nonextensivity in energy (i.e. $E(A+B)=E(A)+E(B)-(1-q) \beta E(A) E(B))$.

The present letter shows that the zero law can hold in the formalism based on the normalization $\operatorname{Tr} \hat{\rho}=1$ and the unnormalized expectation $x=\operatorname{Tr} \hat{\rho}^{q} \hat{x}$. This formalism was proposed by Tsallis and co-workers [2,3] and had great success in many applications [12]. It also has the advantage to give the simplest Legendre transformation. But recently, scientists pay less attention to it due to its peculiar properties such as, among others, $\operatorname{Tr} \hat{\rho}^{q} \hat{1} \neq 1$ and $E(A+B) \neq E(A)+E(B)$ for two independent systems having $\hat{H}(A+B)=\hat{H}(A)+\hat{H}(B)$ |4. These peculiarities make one think that the zeroth law and the first law may be disturbed in this formalism. In the following, we want to show that the zero law can hold, despite the above inequality, in the classic fashion. And the two basic processes of energy change, heat and work, can be interpreted in the same way as in $M B S$ analogy. 


\section{THE ZEROTH LAW}

The maximization of Tsallis entropy' subject to two constraints $\alpha(\operatorname{Tr} \hat{\rho}-1)$ and $\beta\left(\operatorname{Tr} \hat{\rho}^{q} \hat{H}-E\right)$ gives rise to the following distribution function for canonical ensemble [3]

$$
\hat{\rho}=\frac{1}{Z}[1-(1-q) \beta \hat{H}]^{\frac{1}{1-q}}
$$

where

$$
Z=\operatorname{Tr}[1-(1-q) \beta \hat{H}]^{\frac{1}{1-q}}
$$

where the Lagrange multiplier $k \beta=\frac{\partial S}{\partial E}$. Supposing $\hat{\rho}(A+B)=\hat{\rho}(A) \hat{\rho}(B)$ for a total system composed of two correlated subsystems $A$ and $B$ with the same $q$, we obtain,

$$
S(A+B)=S(A)+S(B)+\frac{1-q}{k} S(A) S(B)
$$

and

$$
E(A+B)=E(A) \operatorname{Tr} \hat{\rho}^{q}(B)+E(B) \operatorname{Tr} \hat{\rho}^{q}(A)-(1-q) \beta E(A) E(B)
$$

The relations Eq.(4) and (5) are to be considered as two basic assumptions of the theory. We will establish the zero law on this basis. From Eq.(4), we can write, for a small variation of the total entropy :

$$
\begin{aligned}
d S(A+B) & =\left[1+\frac{1-q}{k} S(B)\right] d S(A)+\left[1+\frac{1-q}{k} S(A)\right] d S(B) \\
& =\left[1+\frac{1-q}{k} S(B)\right] \frac{\partial S(A)}{\partial E(A)} d E(A)+\left[1+\frac{1-q}{k} S(A)\right] \frac{\partial S(B)}{\partial E(B)} d E(B)
\end{aligned}
$$

Because $d S(A+B)=0$, we get

$$
\operatorname{Tr} \hat{\rho}^{q}(B) \frac{\partial S(A)}{\partial E(A)} d E(A)+\operatorname{Tr} \hat{\rho}^{q}(A) \frac{\partial S(B)}{\partial E(B)} d E(B)=0 .
$$

Now from Eq.(2), we easily verify that

$$
\operatorname{Tr} \hat{\rho}^{q}=Z^{1-q}+(1-q) \beta E .
$$

So Eq.(5) becomes

$$
E(A+B)=E(A) Z^{1-q}(B)+E(B) Z^{1-q}(A)+(1-q) \beta E(A) E(B) .
$$

we can write

$$
\begin{aligned}
d E(A+B) & =\left[Z^{1-q}(B)+(1-q) \beta E(B)\right] d E(A) \\
& +\left[Z^{1-q}(A)+(1-q) \beta E(A)\right] d E(B)
\end{aligned}
$$

or, from Eq.(8),

\footnotetext{
${ }^{1}$ Tsallis entropy is given by $S=-k \frac{\operatorname{Tr}_{\hat{\rho}}-\operatorname{Tr}_{\hat{\rho}} q}{1-q},(q \in R)$ [2]
} 


$$
d E(A+B)=\operatorname{Tr} \hat{\rho}^{q}(B) d E(A)+\operatorname{Tr} \hat{\rho}^{q}(A) d E(B)=0
$$

due to $d E(A+B)=0$ for the total system. Comparing Eq.(11) with Eq.(7), we get

$$
\frac{\partial S(A)}{\partial E(A)}=\frac{\partial S(B)}{\partial E(B)}
$$

or $\beta(A)=\beta(B)$. The zeroth law of thermodynamics holds. We can naturally define, as in $M B S$, $\frac{\partial S}{\partial E}=\frac{1}{T}$ and write $T(A)=T(B)$ at equilibrium.

\section{THE FIRST LAW : HEAT AND WORK}

The above discussion suggests that the first law of thermodynamics can be written as before for canonical ensemble :

$$
d E=T d S+f_{i} d x_{i}
$$

From Eq.(8), we easily find Helmoholtz free energy $F$ :

$$
F=E-T S=-\frac{1}{\beta} \frac{Z^{1-q}-1}{1-q}
$$

and

$$
d F=-S d T+f_{i} d x_{i}
$$

It is known that one of the beautiful interpretation of thermodynamics given by statistical mechanics is the understanding of the two kinds of process by which the energy of a system can be changed : heat and work. In $M B S$, transferred heat is interpreted as the energy change related to the probability or population variation $(\operatorname{Tr} \hat{H} d \hat{\rho})$. The work performed by the surroundings on the system is related to the variation of the energy of each state of the system $(\operatorname{Tr} \hat{\rho} d \hat{H})$. We will show that this interpretation can hold in the formalism discussed here.

From the unnormalized expectation mentioned above, we can write

$$
d E=\operatorname{Tr} \hat{H} d \hat{\rho}^{q}+\operatorname{Tr} \hat{\rho}^{q} d \hat{H}
$$

We will show that the first term at the right hand side can be identified to heat $(T d S)$ and the second term to work $\left(f_{i} d x_{i}\right)$.

With the help of Eq.(2), the first term of Eq.(16) can be recast as

$$
\begin{aligned}
\operatorname{Tr} \hat{H} d \hat{\rho}^{q} & =\operatorname{Tr} \frac{1-Z^{1-q} \hat{\rho}^{1-q}}{(1-q) \beta} d \hat{\rho}^{q} \\
& =\frac{1}{(1-q) \beta}\left[\operatorname{Tr} d \hat{\rho}^{q}-Z^{1-q} \operatorname{Tr} \hat{\rho}^{1-q} d \hat{\rho}^{q}\right] \\
& =\frac{1}{(1-q) \beta}\left[d \operatorname{Tr} \hat{\rho}^{q}-Z^{1-q} q \operatorname{Tr} d \hat{\rho}\right] .
\end{aligned}
$$

Considering $\operatorname{Tr} d \hat{\rho}=0$, we get 


$$
\begin{aligned}
\operatorname{Tr} \hat{H} d \hat{\rho}^{q} & =\frac{1}{(1-q) \beta} d \operatorname{Tr} \hat{\rho}^{q} \\
& =\frac{1}{k \beta} d\left[-k \frac{1-\operatorname{Tr} \hat{\rho}^{q}}{1-q}\right] \\
& =T d S \\
& =d Q
\end{aligned}
$$

where $d Q$ is the heat transferred from the surroundings to the system.

New let us see the second term in Eq.(16). We can recast it as

$$
\begin{aligned}
\operatorname{Tr} \hat{\rho}^{q} d \hat{H} & =\operatorname{Tr} \hat{\rho}^{q} \frac{\partial \hat{H}}{\partial x_{i}} d x_{i} \\
& =\frac{1}{Z^{q}} \operatorname{Tr}[1-(1-q) \beta \hat{H}]^{\frac{q}{1-q}} \frac{\partial \hat{H}}{\partial x_{i}} d x_{i} \\
& =-\frac{1}{Z^{q} \beta} \operatorname{Tr}\left\{\frac{\partial}{\partial x_{i}}[1-(1-q) \beta \hat{H}]^{\frac{1}{1-q}}\right\}_{\beta} d x_{i} \\
& =-\frac{1}{\beta} \frac{1}{Z^{q}}\left\{\frac{\partial Z}{\partial x_{i}}\right\}_{\beta} d x_{i} \\
& =-\frac{1}{\beta}\left\{\frac{\partial}{\partial x_{i}} \frac{Z^{1-q}-1}{1-q}\right\}_{\beta} d x_{i} .
\end{aligned}
$$

Considering Eqs.(14) and (15), we obtain finally

$$
\begin{aligned}
\operatorname{Tr} \hat{\rho}^{q} d \hat{H} & =\left\{\frac{\partial F}{\partial x_{i}}\right\}_{\beta} d x_{i} \\
& =f_{i} d x_{i} \\
& =d W
\end{aligned}
$$

where $d W$ is the work performed by the surroundings on the system. So the statistical interpretation of heat and work remains the same in $N S M$ as in $M B S$.

\section{CONCLUSION}

We shown that within the formalism of $N S M$ based on the normalization $\operatorname{Tr} \hat{\rho}=1$ and the unnormalized expectation $x=\operatorname{Tr} \hat{\rho}^{q} \hat{x}$, the zeroth law can hold as in $M B S$. We need not generalized (or physical) temperature, heat and forces [6 8, 10,11] different from that in $M B S$ to keep this basic assumption of thermodynamics. The temperature defined by $T=\frac{\partial E}{\partial S}$ remains the measure of thermodynamic equilibrium. Heat and work are interpreted just as in $M B S$, which seems difficult in the formalisms with normalized expectations. The first law remains the same. This unnormalized formalism finally gives the simplest nonextensive thermodynamic formalism which seems reassuring. This tells us that it is perhaps worthy to evaluate again the unnormalized expectation and to try to find what is hidden behind the peculiarities which remain to be explained. 
[1] G.A. Raggio, Equivalence of two thermostatistical formalisms based on the Havrda-Charvat-Daróczy-Tsallis entropies,arXiv : cond-mat/9908207 (27 Aug.2000) and G.A. Raggio, On equivalence of thermostatistical formalisms,arXiv : condmat/9909161 (10 Sept.2000)

[2] C. Tsallis, J. Statis. Phys.,52(1988)479

[3] E.M.F. Curado, and C. Tsallis, J. Phys. A: Math. Gen.24(1991)L69

[4] C. Tsallis, R.S. Mendes and A.R. Plastino, Physica A,261(1998)534

[5] F. Pennini, A.R. Plastino and A. Plastino, Physica A,258(1998)446

[6] Q.A. Wang, M. Pezeril, L. Nivanen, A. Le Méhauté, Nonextensive distribution function and factorization of the joint probability, Chaos, Solitons \& Fractals,13(2001)131 and e-print, cond-mat/0010294 (19 Oct 2000)

[7] Q.A. Wang, Nonextensive statistics and incomplete information, e-print : cond-mat/0107065 (October 2000)

[8] S. Abe, Physica A,269(1999)403;

[9] S. Abe, Physica A,300(2001)417; S. Abe, S. Martinez, F. Pennini and A. Plastino, Phys. Lett.A,281(2001)126

[10] S. Martinez, F. Pennini, and A. Plastino, Physica A,295(2001)416

[11] S. Martinez, F. Nicolas, F. Pennini, and A. Plastino, Tsallis' entropy maximization procedure revisited, arXiv : physics/0003098 (29 Mar.2000)

[12] C. Tsallis, Entropic nonextensivity : a possible measure of complexity, Chaos, Solitons \& Fractals,13(2001)371 and e-print, arXiv: cond-mat/0010150, (10 Oct.2000) 